\title{
Die Bedeutung kognitiver Prozesse in der Arznei- mittelforschung im 19. Jahrhundert - Das Beispiel Nitroglycerin*
}

Reinhart Schüppel

\section{Summary}

The history of the discovery and development of drugs is replete with examples where chance and «serendipity» have resulted in important advances of knowledge. In the case of nitroglycerin it can be shown that what appears to have been a chance discovery was actually the result of a sequence of selective perceptions by, and cognitive processes in individual researchers. The sources allow insight into various stages of the development of nitroglycerin, starting with the chemical synthesis as an explosive in 1846 and the first use in humans in 1847 to the discovery of a useful coronary drug. Homeopathic medicine contributed significantly to this process. Thus, the history of nitroglycerin is an example of an exchange of knowledge between otherwise separate realms of sectarian and orthodox medicine in the second half of the 19th century.

\section{Zusammenfassung}

In der Geschichte der Entdeckung und Entwicklung von Arzneimitteln lassen sich häufig Ereignisse auffinden, die scheinbar rein zufällig einen wesentlichen Erkenntnisfortschritt auslösten. Am Beispiel der Einführung von Nitroglycerin in die Medizin in der zweiten Hälfte des 19. Jahrhunderts wird gezeigt, dass sich hier der vermeintliche Zufall recht plausibel durch eine Abfolge selektiver Wahrnehmungen und kognitiver Prozesse bei einzelnen

* Der Beitrag enthält wesentliche Ergebnisse eines von der Robert Bosch Stiftung geförderten Forschungsprojekts.

Dr. med. Reinhart Schüppel, Universität Ulm-Klinikum, Abt. Psychotherapie und Psychosomatische Medizin, Robert-Koch-Str. 8, D-89070 Ulm 
Forschern ersetzen lässt. Die relativ gute Quellenlage erlaubt einen guten Einblick in die verschiedenen Stadien der Entwicklung der Substanz Nitroglycerin von der chemischen Synthese als Sprengstoff im Jahr 1846 über den ersten Einsatz am Menschen 1847 bis hin zu seinem Einsatz als Koronartherapeutikum. Der Erkenntnisprozess wurde durch Vertreter der Homöopathie massgeblich beeinflusst. Die Beschäftigung mit den Forschungsbemühungen um Nitroglycerin erlaubt daher zusätzlich einen Einblick in den Wissenstransfer zwischen orthodoxen und sektiererischen Ärzten in der zweiten Hälfte des 19. Jahrhunderts.

\section{Einleitung}

Der Erfolg der modernen Pharmakotherapie beruht ganz wesentlich auf dem Siegeszug der organischen Chemie, die ihre herausragende Funktion als nahezu unerschöpfliche Quelle neuer Medikamente wohl erst in den nächsten Jahren an die Molekularbiologie verlieren wird. Ist heute die Suche nach einem neuen Arzneimittel weitgehend durch systematische Prozesse charakterisiert, so lassen sich für frühere Epochen zahlreiche «Zufälle» auffinden, die den Weg einer Substanz von der Erstbeschreibung bis zur klinischen Anwendung begleitet haben. Bei genauer Betrachtung stellt sich allerdings heraus, dass manches keineswegs rein zufällig passierte, sondern, neben anderen Einflüssen, auch das Resultat spezifischer Wahrnehmungs- und Denkmuster darstellte. Nur deren Unkenntnis lassen solche Ereignisse aus späterer Sicht als Zufall erscheinen ${ }^{1}$.

Das eigentlich als Sprengstoff entwickelte Nitroglycerin wurde gleich durch mehrere solcher Zufälle zu einem wichtigen Medikament. Zu den beteiligten Personen und zum formalen Gang des Wissenstransfers liegen mittlerweile Publikationen vor ${ }^{2}$. Offen bleiben jedoch die Motive und Denkmuster der Protagonisten. Es soll daher der Frage nachgegangen werden, welche kognitiven Prozesse dem «Forscherglück» vermutlich auf die Sprünge geholfen haben. Weil dabei Vertreter der Homöopathie massgebend beteiligt

1 Zur Rolle des Zufalls in der Entwicklung der Arzneimitteltherapie siehe W. Schneider, Geschichte der pharmazeutischen Chemie, Weinheim 1972, zur Arzneimittelgeschichte im 19. Jahrhundert G. Stille, M.H. Bickel, Der Weg der Arznei, Karlsruhe 1994, S. 169-230, 258-259, W.-D. Müller-Jahncke, C. Friedrich, Geschichte der Arzneimitteltherapie, Stuttgart 1996 und F. Chast, Histoire Contemporaine des Médicaments, Paris 1995.

2 Z. B. L.C. Holmes, F.J. DiCarlo, Nitroglycerin: The Explosive Drug, Journal of Chemical Education 9 (1971), S. 573-576, W.B. Fye, Nitroglycerin: A Homeopathic Remedy, Circulation 73 (1986), S. 21-29 und C. Habrich, Nitrat-Geschichte(n). Der Sprengstoff, der auch die Medizin veränderte, Münchner medizinische Wochenschrift 135 (1993), Suppl. 1, S. 5-13. 
waren, soll ausserdem für das 19. Jahrhundert ausführlicher auf die bislang noch zu wenig beachtete Rezeption von Ergebnissen von Aussenseitermethoden durch die etablierte Medizin und umgekehrt eingegangen werden.

\section{Wer hat Interesse an Nitroglycerin?}

Anorganische Nitrate, insbesondere Salpeter, waren spätestens in der Iatrochemie weit verbreitete Arzneistoffe. In der Mitte des 19. Jahrhunderts experimentierten verschiedene Forscher an der Nitrierung organischer Substanzen. Nitroglycerin wurde 1846 vom Italiener Ascanio Sobrero (1818-1888) erstmals beschrieben. Es war für ihn als Chemiker nicht ungewöhnlich, die Eigenschaften einer neuen Verbindung nicht nur im Reagenzglas, sondern auch durch Ingestion einer kleinen Menge zu testen ${ }^{3}$. Sobrero führte dazu aus: «... il suffit d'en tenir une très-petite quantité (ce qu'on peut en prendre en y mouillant légèrement le bout du petit doigt) sur la langue pour en éprouver une migraine assez forte pendant plusieurs heures» ${ }^{4}$.

Die Beobachtungen Sobreros zum Nitroglycerin wurden zunächst von der damaligen orthodoxen Medizin nicht zur Kenntnis genommen, offensichtlich kam keiner ihrer Vertreter auf die Idee, Sprengstoff als ein potentielles Medikament anzusehen. Jedenfalls findet sich zunächst kein Hinwies auf Nitroglycerin in einer regulären medizinischen Publikation. Wieso erkannten die Ärzte die medizinische Bedeutung von Nitroglycerin nicht sofort? Ein möglicher Ansatz, dies recht überzeugend zu erklären, stellt die selektive soziale Wahrnehmung dar.

Soziale Wahrnehmung bezieht sich auf die Identifizierung und Verarbeitung von Informationen aus der sozialen Umwelt. Die Begrenztheit des menschlichen Informationsverarbeitungssystems macht eine fortwährende Zentrierung auf ausgewählte Reize notwendig. Selektionsmechanismen sind Wahrnehmungsbereitschaft und Wahrnehmungshemmung. Als relevant werden allgemein nur solche Informationen empfunden, mit denen der Einzelne oder eine Gruppe «etwas anfangen» kann, die etwa eine Notlage beseitigen helfen oder ganz allgemein eine Grundlage für weitere Aktivi-

3 Zur Prüfung neuer Substanzen durch Geruchs- und Geschmackssinn siehe U. Räth, Zur Geschichte der pharmazeutischen Mineralogie, Braunschweig 1971, S. 140-146.

4 «... es genügt, davon eine sehr kleine Menge auf die Zunge zu nehmen (soviel man braucht, um die Spitze des kleinen Fingers gerade zu benetzen), um einen ziemlich heftigen Kopfschmerz für mehrere Stunden hervorzurufen ...» [Übersetzung durch den Verfasser]. A. Sobrero, Sur Plusieurs Composés Détonants Produits avec l'Acide Nitrique et le Sucre, la Dextrine, la Lactine, la Mannite et la Glycérine, Comptes Rendus des Séances de l'Académie des Sciences 24 (1847), 247-248, S. 247. 
täten bieten. Nichtrelevante Informationen werden dagegen nicht weiter beachtet und bleiben wertlos ${ }^{5}$.

Für «Sprengstoff-Experten» etwa stellte die Publikation von Sobrero eine hochrelevante Information dar, weil sie unmittelbaren Anlass gab, auf eine Erleichterung von Sprengarbeiten zu hoffen, die bis dato unter grossen Gefahren mit Schwarzpulver durchgeführt werden mussten. Allerdings hatten die Chemiker und Ingenieure erhebliche Mühe, das Nitroglycerin so handhabbar zu machen, dass es einerseits bei Herstellung und Transport keinen Schaden anrichtete, andererseits am Einsatzort die nötige Sprengkraft entwickelte. Gelöst wurde das Problem erst Jahre später, als Alfred Nobel (1833-1896) es mit Kieselgur (1867) bzw. Nitrocellulose (1878) zu Dynamit mischte. Nun gab es allerdings doch eine Gruppe in der Medizin, für die die Beobachtungen von Sobrero einen ebenso hohen Informationswert darstellten wie für Sprengstoff-Experten: die homöopathischen Ärzte.

\section{Exkurs 1: Die Aussenseitermethode Homöopathie}

Als Sobrero sein «Knallöl» entdeckt hatte, war Samuel Hahnemann (1755-1843) schon drei Jahre tot. Die von ihm begründete Homöopathie hatte sich zwar in Europa sowie Nord- und Südamerika in einigen Ländern verbreiten können, aber sie war in einer deutlichen Aussenseiterposition geblieben ${ }^{6}$. In Europa war es weder Hahnemann noch einem seiner Schüler gelungen, die Methode etwa dauerhaft an einer Universität zu etablieren. Aber Hahnemann hatte eine medizinische Methodik hinterlassen, die auch nach seinem Tode einer nicht unbeträchtlichen Zahl von Ärzten als Handlungsanleitung diente.

Der zentrale Lehrsatz in Hahnemanns System beinhaltete das Herstellen einer Ähnlichkeitsbeziehung zwischen den Symptomen eines kranken Menschen und denen, die ein Medikament beim Gesunden erzeugen konnte ${ }^{7}$. Wenn es damals durchaus üblich war, dass Ärzte die Wirkung eines neuen Medikaments auch an sich selbst ausprobierten, war es doch Hahnemann vorbehalten gewesen, dies systematisch durchzuführen. Neben dem Studium

5 Einzelheiten zur sozialen Wahrnehmung siehe U. Piontkowski, Soziale Wahrnehmung und Personenwahrnehmung, in F.W. Wilker, C. Bischoff, P. Novak (Hrsg.), Medizinische Psychologie und Medizinische Soziologie, München 1994, S.167-71.

6 Einen Überblick gibt M. Dinges (Hrsg.), Weltgeschichte der Homöopathie, München 1996.

7 Zur Theoriekonstruktion der Homöopathie siehe R. Schüppel, Homöopathie und verwandte Verfahren: Allgemeine Grundlagen. Teil 1: Grundprinzipien, in M. Bühring, F.H. Kemper (Hrsg.), Naturheilverfahren und Unkonventionelle Medizinische Richtungen, Berlin 1994, Kapitel 14.02,1-27, S. 1-5. 
der Krankheiten war für Hahnemann also «der zweite Punkt, des Geschäfts eines ächten Heilkünstlers [...] die Erforschung der, zur Heilung der natürlichen Krankheiten bestimmten Werkzeuge, die Erforschung der krankmachenden Kraft der Arzneien, um, wo zu heilen ist, eine von ihnen aussuchen zu können, aus deren Symptomenreihe eine künstliche zusammengesetzt werden kann, der Haupt-Symptomen-Gesammtheit der zu heilenden natürlichen Krankheit möglichst ähnlich» ${ }^{8}$. Das Beobachten von Medikamentenwirkungen an Patienten hielt er für ungenügend, weil seiner Ansicht nach hierbei nur ein Mischbild aus Arzneieffekt und Krankheitssymptomen resultieren konnte 9 . Aus seiner Sicht blieb daher nur der Weg, Befindensänderungen des Leibes und der Seele, hervorgerufen durch Medikamente, an gesunden Menschen zu studieren ${ }^{10}$.

Er stellte dazu strenge methodische Richtlinien auf, die der Proband, von ihm Prüfer genannt, und der Beobachter, oft war es auch ein und dieselbe Person, zu beachten hatten. Hahnemann forderte, für solche Versuche nur kleinste Mengen der jeweiligen Substanzen zu verwenden. Er war überzeugt, den Probanden damit nicht zu schaden, sondern «... im Gegentheile, dass der Organismus des Prüfenden, durch die mehren Angriffe auf das gesunde Befinden nur desto geübter wird im Zurücktreiben alles seinem Körper Feindlichen von der Aussenwelt her ...» ${ }^{11}$.

Mit erstaunlich grossem Vertrauen übernahmen seine Anhänger diese Sichtweise. Für sie wurde es geradezu selbstverständlich, interessant erscheinende Substanzen, auch wenn diese sonst in der Therapie am Menschen bislang ungebräuchlich waren, entweder selbst einzunehmen oder Familienmitgliedern und Freunden probeweise zu verabreichen.

Einer der treuesten und kreativsten Anhänger Hahnemanns war Constantin Hering (1800-1880) (Abb.1). Aus Sachsen gebürtig, war er nach dem Medizinstudium in Dresden, Leipzig und Würzburg zunächst nach Süd- dann nach Nordamerika ausgewandert und hatte sich schliesslich in Philadelphia niedergelassen. Durch ein Heilungserlebnis am eigenen Leib zum überzeugten Vertreter der Methode geworden, war er in homöopathischen Kreisen durch eine Reihe von Arbeiten früh zu grösserem Ruhm gelangt $^{12}$. Ein Schwerpunkt seiner Tätigkeit war die Testung potentieller

8 S. Hahnemann, Organon der Heilkunst. Textkritische Ausgabe, Heidelberg 1992, \$105.

9 S. Hahnemann (zitiert in Anmerkung 8), \$107.

10 S. Hahnemann (zitiert in Anmerkung 8), \$108.

11 S. Hahnemann (zitiert in Anmerkung 8), \$141

12 Hering und Hahnemann lernten sich nie persönlich kennen, sie hatten aber einen regen Briefwechsel. C. Hering, Chronology of Events in the Life of Constantine Hering, Proceedings of the International Hahnemann Association 39 (1918), 11-37, S. 12. Während des Studiums zog sich Hering eine Sektionsverletzung zu. Die Infektion war zunächst therapieresi- 


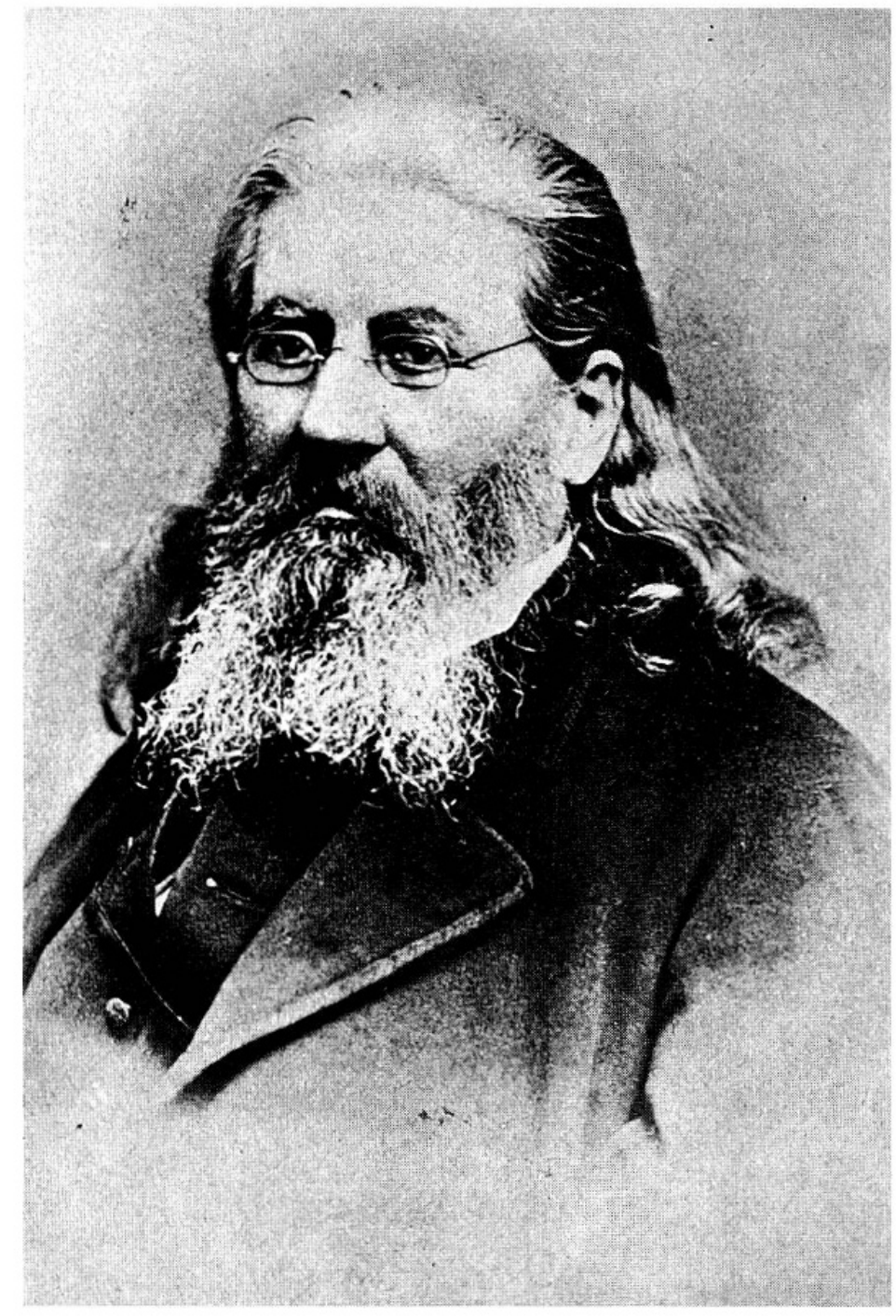

Abb. 1: Constantin Hering (1800-1880). Reproduktion aus: A.M. Eastman, Life and Reminiscences of Dr. Constantine Hering, Philadelphia 1917, S. 13.

Medikamente nach den Regeln, die Hahnemann dafür aufgestellt hatte. In den 1840er Jahren verfügte er bereits über einschlägige Erfahrung, seine Studie mit dem Gift von Lachesis trigonocephalus, der südamerikanischen Klapperschlange, hatte gezeigt, dass er dabei auch vor toxischen Materialien nicht zurückschreckte ${ }^{13}$. Wie Hahnemann verfügte Hering über breite na-

stent, erst nach Einnahme eines homöopathischen Medikaments heilte sie ab. C. Hering, Kurze Bemerkungen. Auszüge aus Constantin Herings Briefen an den Herausgeber, Archiv für die homöopathische Heilkunst 14 (1834), 89-100, S. 92. Zu Einzelheiten über Herings Wirken in den USA siehe R. Schüppel, Constantin Hering (1800-1880) - Ein Akademiker gründet Institutionen, in M. Dinges (Hrsg.), Homöopathie - Patienten, Heilkundige und Institutionen. Von den Anfängen bis heute, Heidelberg 1996, S. 294-315.

13 C. Hering, Wirkungen des Schlangengiftes, zum ärztlichen Gebrauche vergleichend zusammengestellt, Denkschriften der Nordamerikanischen Akademie der homöopathischen Heilkunst, Allentown 1837 und C.B. Knerr, Life of Hering, Philadelphia 1940, S. 140. 
turwissenschaftliche Kenntnisse und verfolgte die Fachliteratur trotz seiner völligen Hinwendung zu der Aussenseitermethode regelmässig.

\section{Homöopathisches Nitroglycerin = Glonoin}

Bei der Durchsicht neuer Publikationen stiess Hering noch im Jahr der Entdeckung auf Sobreros Erstbeschreibung von Nitroglycerin. Für ihn als Angehörigen der medizinischen Aussenseitergruppe der homöopathischen Ärzte stellten die Mitteilungen aus Sobreros Artikel, besonders die deutliche Beschreibung des Effekts auf den menschlichen Organismus nach Einnahme einer nur geringen Substanzmenge hochrelevante Informationen dar. «Dieses Öl musste ich haben, es koste was es wolle. Wenn es mit solcher Bestimmtheit Kopfweh macht, so muss es dasselbe auch mit gleicher Bestimmtheit heilen. Wenn es aber bei Chemikern heftiges Kopfweh macht man bedenke, was das heisst: bei Chemikern! die gegen eine Menge der dem Körper fremdartigsten Stoffe callös werden müssen, - wenn es nicht nur Einem oder dem Andern, sondern Mehren und mehrmals macht, und zwar in «sehr kleinen Mengen», was muss das für einen überwiegenden Einfluss haben auf den Menschen!» ${ }^{15}$. Er fand also offensichtlich in Sobreros Arbeit nicht nur die Darstellung eines neuen Sprengstoffes, sondern die (unbeabsichtigte) Beschreibung einer medizinischen Substanzprüfung am Gesunden, die die Homöopathie zur Haupterkenntnisquelle von Arzneiwirkungen erklärt hatte.

Hering führte in den Jahren 1847-1849 eine erste systematische Testserie mit insgesamt 21 Probanden durch. Nitroglycerin wurde dazu in Dosen von einem fünftausendstel Tropfen bis zu zehn Tropfen eingenommen ${ }^{15} .1849$ publizierte er erste Ergebnisse mit dem Stoff, den er Glonoin(e) nannte ${ }^{16}$. In den Publikationen wies Hering besonders auf die nach der Einnahme auftretenden Kopfschmerzen hin, sowie auf eine fast regelmässig zu beobach-

14 C. Hering, Glonoin oder Nitro-Glycerin, in C. Hering, Amerikanische Arzneiprüfungen, Leipzig 1853, 21-140, S. 22.1857 erschien ein unveränderter Nachdruck.

15 Die ersten Prüfungen wurden mit unverdünntem Nitroglycerin durchgeführt. Das Teilungsverhältnis ergibt sich durch die Benetzung von 5000 Milchzuckerkügelchen durch einen Tropfen. Neben der Einnahme von kleinen Mengen unverdünnter Substanzen war bei homöopathischen Arzneiprüfungen und in der Therapie in der Mitte des 19. Jahrhunderts das Verabreichen von 1:100 mehrmals verdünnten und verschüttelten Arzneien üblich, Glonoin wurde bis zur 30. Verdünnungsstufe geprüft. C. Hering, Glonoine, British Journal of Homeopathy 7 (1849), 413-421, S. 413-414.

16 Das Kunstwort Glonoin(e) ist zusammengesetzt aus GLO (Oxyde of Glycyl), NO (Nitric acid) und der Nachsilbe INE, im Deutschen ohne «e». C. Hering, Glonoine, Quartely Homeopathic Journal 1 (1849), 440-451, S. 441. 
tende Tachykardie. Damit war Nitroglycerin in die Medizin eingeführt, jedenfalls in eines ihrer «sektiererischen» Subsysteme. Grundlage des Prozesses war ein Analogieschluss gewesen, hier in Form der homöopathischen Ähnlichkeitsregel.

Nach weiteren Versuchen publizierte Hering 1853 schliesslich eine systematische Übersicht, die auf über 140 Beobachtungen an Gesunden, einigen Patienten und interessanterweise auch an drei Katzen und einem Frosch beruhten (Abb. 2). Innerhalb des homöopathischen Lagers fand das neue Medikament auch international rasch Eingang in einschlägige Zeitschriften und Standardwerke der Materia Medica ${ }^{17}$. Wenn Nitroglycerin also in der Homöopathie ein gebräuchliches Medikament geworden war, hatte man dort schon seine Bedeutung als Koronartherapeutikum erkannt?

\section{Exkurs 2: Angina pectoris}

Das heute als Angina pectoris definierte Krankheitsbild lässt sich in vielen älteren Beschreibungen wiederfinden ${ }^{18}$. Erst William Heberden (17101801) aber lieferte 1768 eine umfassende und explizite klinische Darstellung der Erkrankung, die noch lange Zeit später als Heberdensche Angina pectoris bezeichnet wurde. War seit dem Ende des 18. Jahrhunderts somit das klinische Bild einigermassen klar umrissen, so machte die Aufklärung der Pathogenese erheblich mehr Schwierigkeiten. Die Arbeiten von Edward Jenner (1749-1823) und Caleb Parry (1755-1822) etwa wiesen schon früh auf eine Beziehung zwischen Koronarerkrankung und Schmerz hin, aber eine umfassende und weitgehend widerspruchsfreie Theorie der koronaren Herzkrankheit sollte erst im 20. Jahrhundert gelingen ${ }^{19}$.

17 C.J. Hempel, New Homeopathic Pharmacopoeia and Posology, New York 1850, S. 327-328, J. Buchner, Homöopathische Arznei-Bereitungslehre, zweite sehr vermehrte Auflage, München 1852, S. 301-303, R.E. Dudgeon, On the Pathogenetic and Therapeutic Action of Glonoine, British Journal of Homoeopathy 11 (1853), S. 268-292, Anonymus, Glonoine, ein neues Arzneimittel ..., Allgemeine homöopathische Zeitung 42 (1852), S. 189, V. Meyer, Ein Collectivbild der Glonoinwirkungen, Allgemeine homöopathische Zeitung 64 (1862), S. 49-52, 57-60; W.E. Payne, Prüfung des Glonoin, Allgemeine homöopathische Zeitung 63 (1861), S. 119, 128; W. Böricke, Glonoin, Allgemeine homöopathische Zeitung 132 (1896), S. 9-12. In Pharmakopöen der regulären Medizin taucht Nitroglycerin erst gegen Ende des 19. Jahrhunderts bzw. (z. B. in Deutschland) zu Beginn des 20. Jahrhunderts auf. C. Habrich (zitiert in Anmerkung 2), S. 11.

18 Siehe hierzu J.O. Leibowitz, The History of Coronary Heart Disease, London 1970 und C. Lawrence, «Definite and Material»: Coronary Thrombosis and Cardiologists in the 1920s, in C.E. Rosenberg, J. Golden (Hrsg.), Framing Disease. Studies in Cultural History, New Brunswick NJ 1992, S. 50-82.

19 J.O. Leibowitz (zitiert in Anmerkung 18), S. 104-145. C. Lawrence (zitiert in Anmerkung 18), S. $60-61$. 


\section{AMERIKANISCHE}

\section{ARZNEIPRÜFUNGEI.}

\section{VORARBEITEN whitent}

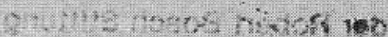

ZUR

\section{ARZNEILEHRE ALS NATURWISSENSCHAFT}

VON

\section{CONSTANTIN HERING.}

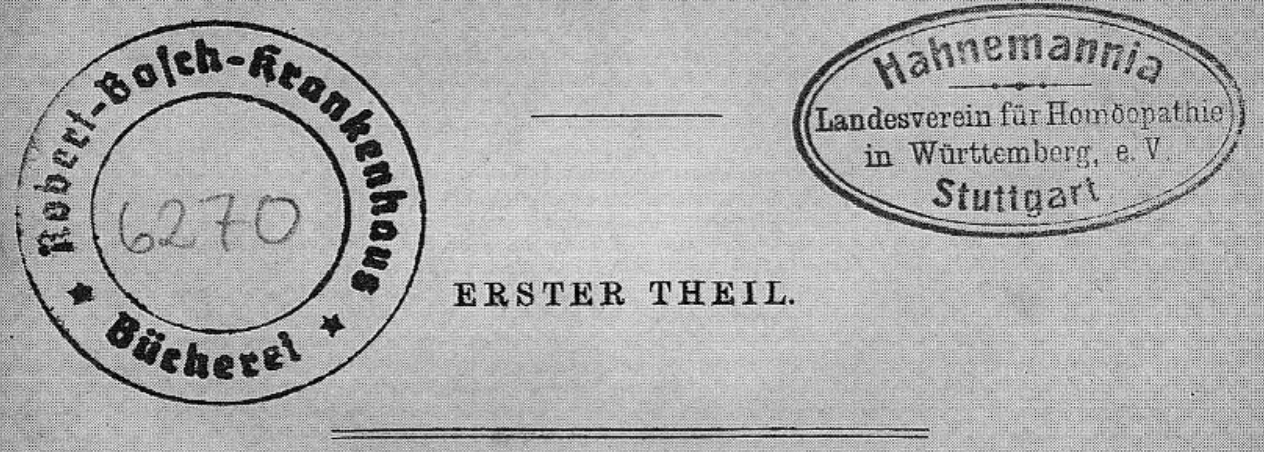

\section{LUIPZIG \& HIIDELBERG,}

C. F. WINTER'SCHE VERLAGSHANDLUNG.

1857.

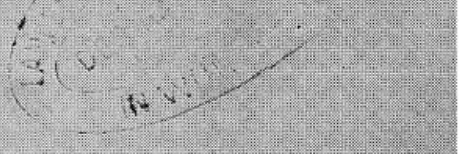

Abb. 2: Amerikanische Arzneimittelprüfungen (Nachdruck von 1857). Der Band enthält die erste umfassende Beschreibung der Wirkungen von Nitroglycerin auf den menschlichen Organismus. Abdruck mit freundlicher Genehmigung des Instituts für Geschichte der Medizin der Robert Bosch Stiftung, Stuttgart. 
Die homöopathischen Ärzte des 19. Jahrhunderts hatten, weil sie in der Mehrzahl Absolventen regulärer Ausbildungsstätten waren, eine genaue Kenntnis des Beschwerdebildes der Angina pectoris ${ }^{20}$. Über deren Ursache wussten sie nicht mehr, aber vermutlich auch nicht weniger als ihre orthodox praktizierenden Kollegen, wenn sie sich denn für «herkömmliche» Krankheitsursachen interessierten, bezüglich dieser Fragestellung gab es erheblichen Streit innerhalb der Homöopathie ${ }^{21}$. Auf jeden Fall waren die homöopathischen Ärzte, gleich welchem Lager sie angehörten, Spezialisten für «Symptomatologie», insbesondere dafür, Arzneiprüfungssymptome und klinisch beobachtete subjektive und objektive Symptome zur Deckung zu bringen.

Homöopathische Ärzte lehnten zwar oft die Verordnung ihrer Medikamente nach klinischen Diagnosen ab, weil damit der individuellen Symtomatik nicht genügend Rücksicht geschenkt werde, jedoch wurde weithin akzeptiert dass einzelne Medikamente eine besondere Affinität zu bestimmten Organsystemen aufwiesen. Glonoin hatte den Ruf, ein hervorragendes Kopfschmerzmittel zu sein ${ }^{22}$. Dieses Symptom, heute als Nitratkopfschmerz gut bekannt, hatte alle Prüfer vom unfreiwilligen Erstbeschreiber Sobrero über Hering bis hin zu allen späteren Beobachtern sowohl von der Zahl der verschiedenen Sensationen als auch von ihrer Intensität her am stärksten beeindruckt. Waren aber bei den Prüfungen an Gesunden keine Herzbeschwerden aufgetreten?

Bei genauer Durchsicht der detailliert erhaltenen Beschreibungen von Hering finden sich Symptome wie «grosse Furcht bei dem Gefühle, als wäre die Brust wie zusammengeschraubt (Nr. 3)», «Zusammenziehen der ganzen Brust als würden Ketten herumgelegt und immer mehr zusammengeschnürt (Nr. 604)» oder «lanzinierender Schmerz aus der Herzgegend nach dem Rücken zu, bis zwischen die Schultern (Nr. 620)» die sich wie eine lehrbuchmässige Beschreibungen der Angina pectoris anhören ${ }^{23}$. Als klinische

20 C. Hering, The Guiding Symptoms of our Materia Medica, Vol. V, Philadelphia 1887, S. 422 und S. Lilienthal, Therapeutics of Angina Pectoris, North American Journal of Homeopathy 23 (1874), S. 103-110.

21 Beispielhaft sei hier der Streit zwischen Constantin Hering und Adolph Lippe (1812-1888) um die Errichtung eines Lehrstuhls für Pathologie am Homoeopathic Medical College of Pennsylvania in Philadelphia genannt. Lippe war der Ansicht, dass es gegen die reine Lehre der Homöopathie verstosse, zukünftige Ärzte in Pathologie zu unterrichten. Hering dagegen unterstützte nachhaltig die Ausbildung der Medizinstudenten in Grundlagenfächern. N. Rogers, The Proper Place of Homoeopathy, Pennsylvania Magazine of History and Biography 108 (1984), 182-186, S.184.

22 C. Hering, Glonoine, a New Medicine for Headache \& c., American Journal of Homeopathy 4 (1849), S. 3-5.

23 C. Hering (zitiert in Anmerkung 14), S. 93-126. Die in Klammern angegebenen Zahlen geben die dortigen Symptomnummern wieder. 
Indikationen für Nitroglycerin nannte Hering, unter Berücksichtigung individueller Besonderheiten, «Sonnenstich, Kinetose, Digitalisintoxikation, Meningitis und Epilepsie» aber auch «Brustcongestion» ${ }^{24}$. Hat Hering damit Angina pectoris gemeint? Möglich ist dies, es fragt sich aber, warum er den Ausdruck, der ihm geläufig war, dann nicht benutzt hat. Jedenfalls führte Samuel Lilienthal (1815-1891), ein von München nach Amerika emigrierter Absolvent des New York Homoeopathic Medical College 1874, mit ausdrücklichem Bezug auf Hering, unter 34 homöopathischen Medikamenten zur Therapie der Angina pectoris Glonoin nicht auf ${ }^{25}$.

Veit Meyer (1815-1872), ein führender Leipziger Homöopath, zeitweilig auch Schriftleiter der Allgemeinen Homöopathischen Zeitung, empfahl den Einsatz des Medikaments bei einer Reihe von Herzerkrankungen, wiederum wird die Angina pectoris nicht explizit erwähnt ${ }^{26}$. Erst gegen Ende des 19. Jahrhunderts lassen sich dann in homöopathischen Standardwerken Hinweise auf Glonoin zur Behandlung von Angina pectoris nachweisen, teilweise unterscheiden die Autoren zwischen der homöopathischen Wirkung des Medikaments z. B. bei Kopfschmerzen und einer allopathischen bei Angina pectoris ${ }^{27}$. Hier lässt sich aber nicht mehr differenzieren, inwieweit mittlerweile vorhandene Kenntnisse aus der Schulmedizin eingeflossen sind.

Homöopathische Ärzte stellten immerhin vor ihren regulär praktizierenden Kollegen eine Beziehung von Nitroglycerins zu Herzerkrankungen her. Von Carroll Dunham (1828-1877) stammen zwei Kasuistiken aus dem Jahr 1853 von Herzpatienten, die mit Glonoin behandelt wurden ${ }^{28}$. Dass die Substanz in der Homöopathie aber als «Herzmittel» gehandelt worden wäre, lässt sich nicht ohne weiteres behaupten. Wie später noch ausgeführt, übernahmen die Schulmedizin Nitroglycerin zunächst praktisch unverändert mit seinen aus der Homöopathie bekannten Indikationen, zu denen die Angina pectoris nicht in erster Linie zählte.

Interessanterweise lagen auch später, nachdem sich die konventionelle Medizin ausgiebiger mit Nitroglycerin beschäftigt hatte, die Vorstellungen homöopathischer und regulärer Ärzte zum Wirkmechanismus der Substanz nicht weit auseinander. Meyer beispielsweise erklärte 1862 die Wirkung von Glonoin über einen «Angriff nur auf das Centralorgan des Kreislauf, oder um physiologischer zu sprechen auf die motorischen Herznerven». Es rief nach seiner Ansicht folglich «eine zum Theil sehr stürmische Hyperämie oder

24 C. Hering (zitiert in Anmerkung 14), S. 139-140.

25 S. Lilienthal (zitiert in Anmerkung 20), S. 106.

26 V. Meyer (zitiert in Anmerkung 17), S. 58.

27 C. Hering (zitiert in Anmerkung 20), S. 422, J.H. Clarke, A Dictionary of Practical Materia Medica, Vol. II, London 1902, S. 824. W. Böricke (zitiert in Anmerkung 17), S. 11.

28 C. Hering (zitiert in Anmerkung 20), S. 407. 
active Congestion der über dem Zwergfell gelegenen Organe vor Augen», verursacht durch «die Erzeugung eines Sturms im Gefässsystem» ${ }^{29}$. William Böricke (1849-1929), einer der wichtigsten Exponenten der amerikanischen Homöopathie an der Westküste, vertrat die Ansicht, dass Nitroglycerin die Tätigkeit von Herz und Arterien steigere. Nach seiner Ansicht war der eigentliche Angriffspunkt aber die Medulla oblongata, hier sollten Vagus und vasomotorisches Zentrum beeinflusst werden, eine Vasodilatation und Anregung der Herztätigkeit wären dann die Folge ${ }^{30}$. Diese Theorie ist aus heutiger Sicht unhaltbar, unterschied sich jedoch nicht wesentlich von den Vorstellungen, die die damalige Schulmedizin von der Nitroglycerinwirkung hatte ${ }^{31}$.

\section{Nitroglycerin als Angina pectoris-Mittel}

Wie Nitroglycerin nun vom Aussenseiterzirkel in die etablierte Medizin gelangte, lässt sich relativ gut nachvollziehen, weil hier eine überschaubare Zahl von Beteiligten jeweils explizit festgehalten hat, wo Informationen die Wahrnehmungsschwelle überwinden konnten und welche weitergehenden Überlegungen daraus folgten. Obwohl Herings Verhältnis zur damaligen konventionellen Medizin durch ein Überlegenheitsgefühl und die entschiedene Ablehnung ihrer therapeutischen Prinzipien gekennzeichnet war $^{32}$, blieb er aufgrund seines naturwissenschaftlichen Interesses ambivalent und war offensichtlich neugierig, was die «alte Schule» mit der Substanz anfangen würde. Er stellte dem Medizinstudenten William Jackson Nitroglycerin aus seinem Arzneifundus für eine Dissertation am regulären Jefferson Medical College in Philadelphia zur Verfügung. Die Publikation der Ergebnisse der Arbeit 1849 im Medical Examiner enthielt allerdings nur wenige schlechtbeschriebene klinische Beobachtungen, die offensichtlich nicht geeignet waren, Interesse bei Kollegen zu wecken ${ }^{33}$.

29 V. Meyer (zitiert in Anmerkung 17), S. 50, 59.

30 W. Böricke (zitiert in Anmerkung 17), S. 11.

31 Dargestellt z. B. bei A.J. Minor, Tri-Nitro-Glycerine. Its Physiological Effects and Therapeutical Indications, American Psychological Journal 3 (1876), 103-111, S. 110 und J.O. Leibowitz (zitiert in Anmerkung 18), S. 105-176.

32 «Was konnte die alte Schule mit Sobrero's Knallöl anfangen? ... Vielleicht alten Leuten den Puls geschwinder machen? Das würde eine kurze Freude geben. Vielleicht bei Herzkranken wenn's zum Tode geht? Das würde ein kurzes Leiden geben ...». C. Hering (zitiert in Anmerkung 14), S. 27-28.

33 Hering (zitiert in Anmerkung 14), S. 40. Jackson gibt als Bezugsquelle des Nitroglycerins nur Herings Chemiker an. Anders als Hering hatte Jackson erhebliche Probleme, Freiwillige für die Experimente am Menschen zu finden. W.F. Jackson, On the Toxical and other Properties of a Substance Analoguos to Gun Cotton, Medical Examiner (Philadelphia) 5 (1849), 
Ganz anders dagegen als neun Jahre später der englische Arzt Alfred G. Field in der angesehenen Medical Times and Gazette über Nitroglycerin berichtete. Er war durch einen nicht namentlich bekannten homöopathischen Arzt aufgrund eines gemeinsam durchgeführten Selbstversuches auf die Substanz aufmerksam geworden. Nach einigen Tierxperimenten entschloss er sich, Nitroglycerin an Patienten einzusetzen. Die therapeutischen Empfehlungen, die daraus entstanden, blieben ganz dicht an denen, die aus der Homöopathie bekannt waren: Kopfschmerzen, Neuralgien und abdominelle Spasmen $^{34}$.

Angeregt durch Fields Publikation legten Henry W. Fuller und George Harley, zwei Kollegen aus London, kurze Zeit später eigene Erfahrungen vor. Auch Sie führten Selbst- und Tierversuche durch. In einer Dosis, die etwa der einmaligen Verdünnungsstufe 1:100 entspricht, fanden sie zwar nur einen Teil der von Field beschriebenen «homöopathischen» Phänomene wieder, Harley war jedoch über die geringe Substanzmenge, die noch zu Symptomen führte, so überrascht, dass er bemerkte: «....and I must candidly admit, that if all infinitesimal doses are equally potent, my ideas of homoeopathy require to undergo a radical change ${ }^{35}$. Field publizierte ein Jahr später Kasuistiken von Patienten mit Neuralgien, die kleine Mengen des Medikaments unverdünnt erhielten. In dieser Publikation wird letztmals der Ausdruck Glonoin benutzt, in den folgenden schulmedizinischen Publikationen findet sich nur noch die Bezeichnung Nitroglycerin ${ }^{36}$.

Bis zu diesem Zeitpunkt hatte die reguläre Medizin dem homöopathischen Wissen also noch keine wesentlich neuen Erkenntnisse hinzugefügt. Auch die in den 1860er Jahren durchgeführten Untersuchungen durch namhafte Physiologen in den USA und in Deutschland bestätigten lediglich alte Beobachtungen $^{37}$, bezüglich des Wirkmechanismus bewegte man sich noch

279-283, S. 279. Die zweite Erwähnung von Nitroglycerin in einem nicht-homöopathischen Journal findet sich 1854. Dort wurden Äusserungen Herings insbesondere zum Sprengstoffcharakter der Substanz so zusammengefügt, dass sich der Eindruck ergeben musste, hier habe es ein Verrückter auf das Leben seiner Patienten abgesehen. L. Kofler, Ueber Glonoin oder Nitroglycerin; zugleich als Probe homöopathischer Charlatanerie, Vierteljahresschrift für praktische Pharmacie 3 (1854), S. 225-231.

34 A.G. Field, On the Toxical and Medical Properties of Nitrate of Oxide of Glycyl, Medical Times and Gazette 16, March 20 (1858), S. 291-292.

35 H.W. Fuller, Nitro-Glycerine - Glonoine, Medical Times and Gazette 16, April 3 (1858), S. 356, G. Harley, Nitro-Glycerine - Glonoine, Medical Times and Gazette 16, April 3 (1858), S. 356-357.

36 A.G. Field, Nitro-Glycerine or Glonoine, Medical Times and Gazette 18, April 2 (1859), S. 339-341.

37 Beispielsweise J.F.H. Albers, Die physiologische und therapeutische Wirkung des Nitroglycerins, Deutsche Klinik, Zeitung für Beobachtungen aus deutschen Kliniken und Krankenhäusern 42 (1864), 405-408 oder A.J. Minor (zitiert in Anmerkung 31), S. 103-111. 
Influcnce of Nitrite of A myl on the Pulsc.

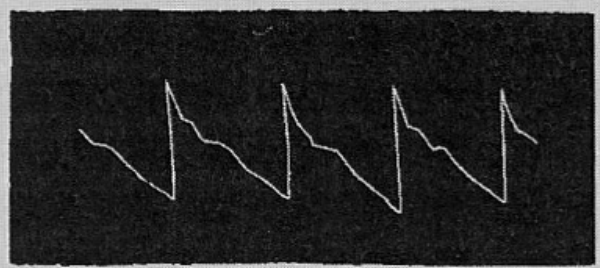

No. 1-Before inhalation.

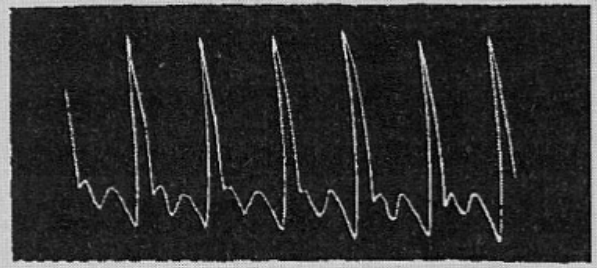

Nio. 2. - One minute after inhalation.

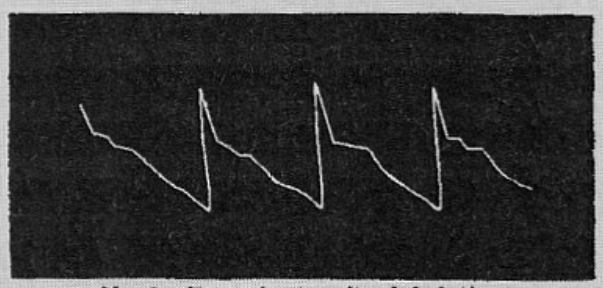

No. 3. - Two minutes after inhalation.
Infuence of Nitro-Glycerine on the Pulse.

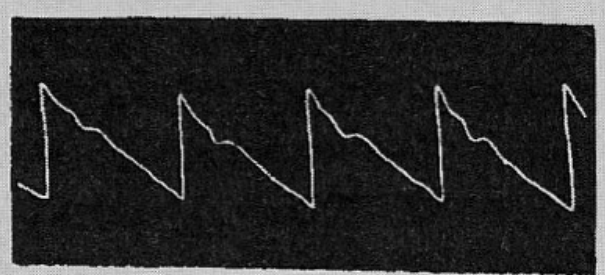

No. 1.-Before dose.

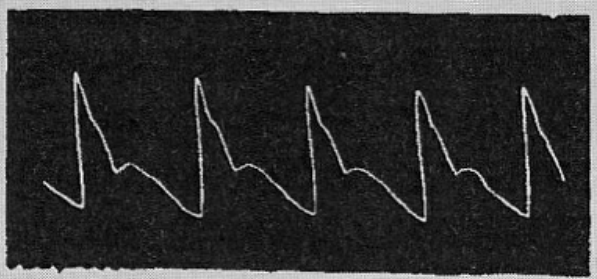

No. 2. $\mathrm{T}$ wo minutes after dose.

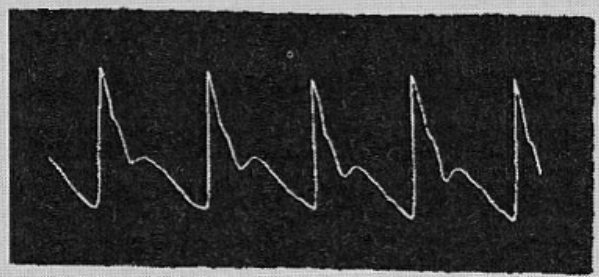

Nor 3. - Eight minutes alter dose.

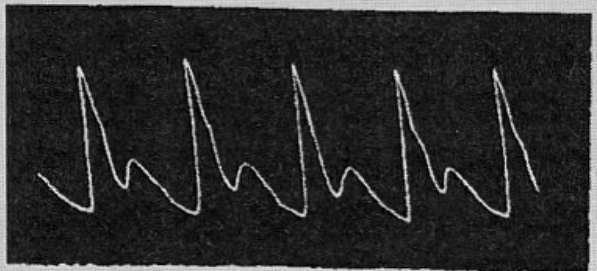

No \& Nine minutes aflor dowe

Abb. 3: Registrierung der Pulskurven von Amylnitrit und Nitroglycerin. Die Ähnlichkeit der Wirkung der beiden Substanzen brachte William Murrell auf die Idee, Nitroglycerin bei Angina pectoris einzusetzen. Reproduktion aus: W. Murell (zitiert in Anmerkung 42), S. 114.

lange Zeit auf bekannten Bahnen ${ }^{38}$. Die Vorschläge zur Indikation für Nitroglycerin umfassten so unterschiedliche Krankheiten wie Asthma, Seekrankheit, Keuchhusten, Migräne, Apoplexie und Nephritis, Krankheiten, deren Pathophysiologie man noch nicht verstand ${ }^{39}$. Bis in die 1870 er Jahre war in der Schulmedizin ausserdem umstritten, ob Nitroglycerin überhaupt ein hilfreiches Medikament oder nicht vielmehr ein Gift sei, welches man tunlichst aus der Therapie eliminieren solle ${ }^{40}$.

38 M. Neuburger, Die historische Entwicklung der experimentellen Gehirn- und Rückenmarksphysiologie vor Flourens, Stuttgart 1897, S. 252-262.

39 W.B. Fye (zitiert in Anmerkung 2), S. 25-27.

40 A.J. Minor (zitiert in Anmerkung 31), S.103, fasst in seiner Arbeit den Stand der damaligen Diskussion zusammen. Er selbst war der Überzeugung, dass Nitroglycerin ein wichtiges Medikament darstelle. 
Seinen Siegeszug als Medikament gegen Angina pectoris verdankt Nitroglycerin dann ein weiteres Mal einer hochselektiven Wahrnehmung, die sich wiederum an einer Analogiebeziehung orientierte. 1867 hatte der schottische Arzt Thomas Lauder Brunton (1844-1916) Amylnitrit in die Therapie der Angina pectoris eingeführt ${ }^{41}$. William Murrell (1853-1912), Arzt und Physiologe am Londoner Westminster Hospital, war mit dieser Behandlung aus der Literatur vertraut, als er sich in den 1870er Jahren für Nitroglycerin zu interessieren begann. Er hatte die Arbeiten von Field, Fuller und Harley gelesen und führte eigene Versuche mit Nitroglycerin an Gesunden und an Patienten durch.

Die Beobachtungen, die er dabei festhalten konnte, erinnerten ihn frappant an die experimentellen Daten, die mit dem hochwirksamen Amylnitrit erzielt worden waren. Da Nitroglycerin in seiner physiologischen Wirkung offenbar weitgehend mit der von Amylnitrit übereinstimmte, setzte Murrell es bei Angina pectoris ein: «From a consideration of the physiological action of the drug, and more especially from the similarity existing between its general action and that of nitrite of amyl, I concluded that it would probably prove of service in the treatment of angina pectoris ... $\gg^{42} .1879$ publizierte er dann seine Erfahrungen mit Nitroglycerin anhand dreier Kasuistiken von Angina pectoris-Patienten ${ }^{43}$. Die Pulskurven, die Murrells Analogieschluss ausgelöst hatten, sind in der Abbildung 3 wiedergegeben. Damit war Nitroglycerin in den ärztlichen Alltag und in die Forschungslabors der Schulmedizin eingebracht.

41 B.T. Lauder, On the Use of Nitrite of Amyl in Angina Pectoris, Lancet II (1867), S. 97-98. Auch Hering befürwortete später den «palliativen» Einsatz von Amylnitrit bei Angina pectoris. C. Hering, Euthanasia, North American Journal of Homeopathy 9 (1879), 257-265, S. 265.

42 W. Murrell, Nitro-Glycerine as a Remedy for Angina Pectoris, Lancet I (1879), 80-81, 113-115, 151-152, 225-227, S. 113.

43 W. Murrell (zitiert in Anmerkung 42). 